\title{
Cutaneous dermoid cyst: Cytokeratin and filaggrin expression suggesting differentiation towards follicular infundibulum and mature sebaceous gland
}

\author{
ICHIRO KUROKAWA ${ }^{1}$, KEISUKE NISHIMURA ${ }^{1}$, ARATA HAKAMADA ${ }^{1}$, KEN-ICHI ISODA ${ }^{1}$, \\ KEI-ICHI YAMANAKA ${ }^{1}$, HITOSHI MIZUTANI ${ }^{1}$ and AIRO TSUBURA ${ }^{2}$ \\ ${ }^{1}$ Department of Dermatology, Mie University Graduate School of Medicine, Tsu, Mie 514-8507; \\ ${ }^{2}$ Department of Pathology, Kansai Medical University, Moriguchi, Osaka 570-8506, Japan
}

Received February 20, 2006; Accepted April 10, 2006

\begin{abstract}
We experienced two cases of cutaneous dermoid cysts (DC). To elucidate the histogenesis of DC, we have studied cytokeratin (CK) expression in DC using ten different anti-keratin antibodies against CK1, 7, 8, 10, 14, 15, 16, 17, 18 and 19, and anti-filaggrin (filament aggregating protein) antibody. In the cyst wall of DC, CK1 and 10 were expressed in suprabasal layer, and CK14 was limited to the basal layer. In sebaceous gland-like structures, CK14 was detected in sebaceous acinus, and CK17 was detected in sebaceous duct. The other CKs were not detected. Filaggrin was intensely detected in the granular layer in the cyst wall of DC. CK expression profile of DC was similar to follicular infundibulum and sebaceous gland. These results suggested that DC differentiates towards follicular infundibulum and mature sebaceous gland.
\end{abstract}

\section{Introduction}

Cutaneous dermoid cysts result from the sequestration of cutaneous tissue along lines of embryonic fusion (1). DC in the periorbital area, midline of the neck, nasal root, forehead, mastoid area and the scalp have been reported (2). DCs of the auricle are very rare (3). The histogenesis of DC still remains unclear.

$\mathrm{CK}$ is an essential marker to evaluate the origin of epithelial tumors (4). Besides CK, filament aggregating protein (filaggrin), a histidine-rich phosphorylated basic protein of major constituents of keratohyaline granules, is a marker of terminal differentiation of epidermis (5). No immunohistochemical study of CK and filaggrin in DC has been reported.

Correspondence to: Dr Ichiro Kurokawa, Department of Dermatology, Mie University Graduate School of Medicine, 2-174 Edobashi, Tsu, Mie 514-8507, Japan

E-mail: kuroichi@clin.medic.mie-u.ac.jp

Key words: cutaneous dermoid cyst, cytokeratin, filaggrin
To elucidate the histogenesis of DC, we studied CK and filaggrin expression in DC using ten different anti-keratin antibodies against CK1, 7, 8, 10, 14, 15, 16, 17, 18 and 19, and anti-filaggrin antibody.

\section{Materials and methods}

The patient, a 48-year-old male had DC located on the left postauriclar area from at birth. The other patient, a 31-yearold female developed DC on the right postauriclar area ten years ago. The lesions were surgically excised. Each specimen was formalin-fixed, paraffin-embedded, stained with hematoxylin and eosin, and serially-cut sections were used for the immunohistochemical study. The anti-keratin antibodies used were as follows: 34ßB4 (CK1) (6), LHP1 (CK10) (7), LL002 (CK14) (7), LHK15 (CK15) (8), LL025 (CK16) (7), E3 (CK17) (7), 5D3 (CK18) (7) and b170 (CK19) (9) (all from Novocastra Laboratories Ltd., Newcastle-upon-Tyne, UK).

The immunohistochemical study was carried out with the labeled streptoavidin-biotin method (LSAB, Dako, Carpenteria, CA, USA) was performed as previous study (10). Normal skin from the auricle served as controls.

\section{Results}

Hematoxylin and eosin staining. Histopathological findings of the cases were similar. The cyst wall of DC was located in the deep dermis. Cyst wall was partly ruptured, resulting in the fibrosis with vellus hairs. The cyst wall was formed with keratinizing epithelium in granular layers. The keratinizing epithelium partly projected into the lumen-like crenulation. Thick laminated corneocytes were observed. In the vicinity of the cyst wall, sebaceous gland-like structure was observed (Fig. 1).

Immunohistochemical findings. $\mathrm{CK}$ and filaggrin expression in normal pilosebaceous unit, DC and steatocystoma multiplex (10) was summarized in Table I.

The epithelial components of DC were divided into two structures: cyst wall and sebaceous gland-like structure (SGLS). CK1 (Fig. 2) and CK10 were expressed in suprabasal 


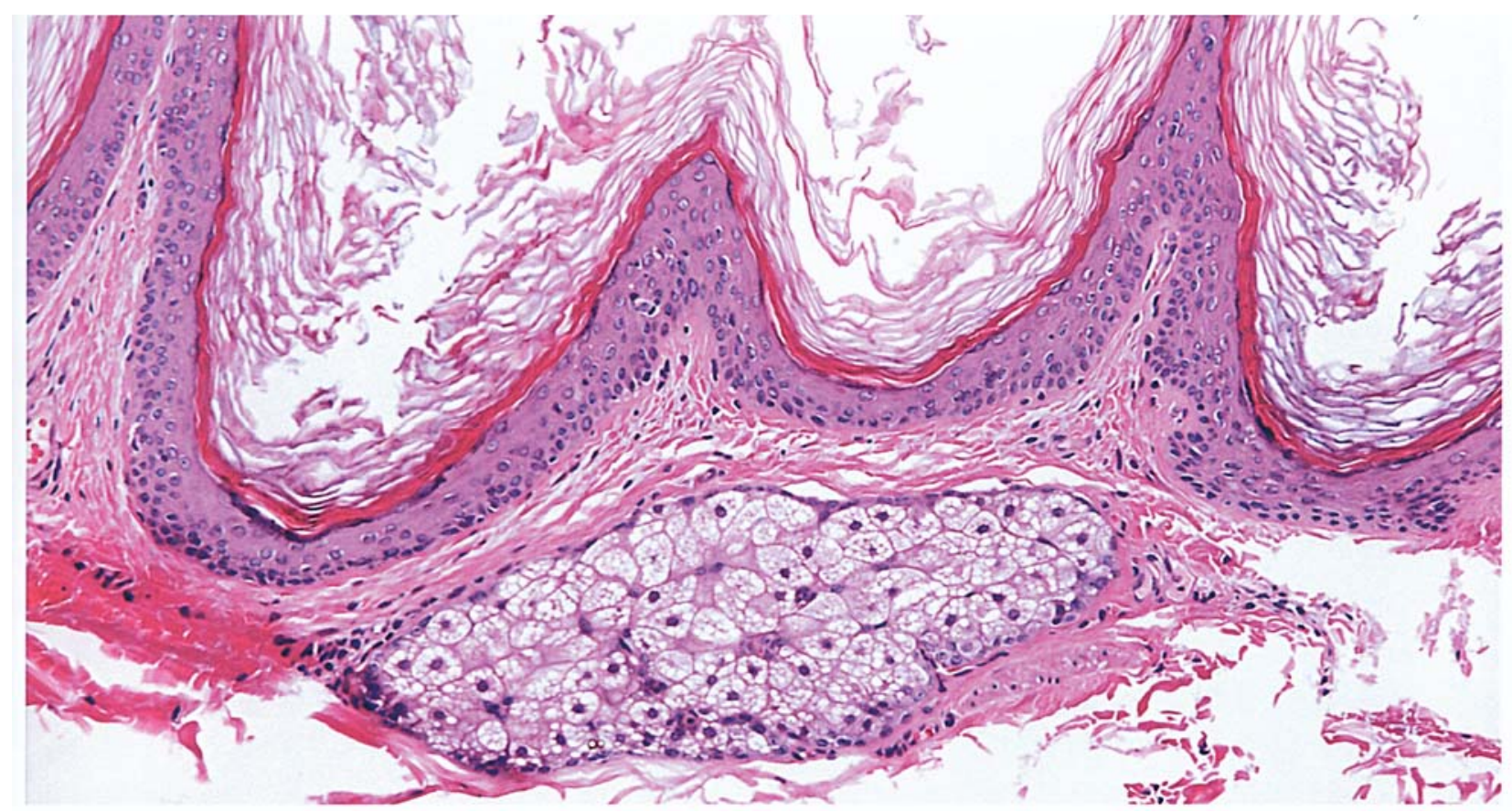

Figure 1. The crenulated cyst wall with keratohyaline granules projected into the lumen. In the vicinity of the cyst wall, sebaceous gland-like structure is observed (hematoxylin and eosin staining; original magnification $\mathrm{x} 100$ ).

Table I. CK and filaggrin expression in normal skin, dermoid cyst and steatocystoma multiplex. ${ }^{a}$

\begin{tabular}{|c|c|c|c|c|c|c|c|c|c|}
\hline & \multicolumn{4}{|c|}{ Normal skin } & \multicolumn{3}{|c|}{ Dermoid cyst } & \multicolumn{2}{|c|}{$\begin{array}{l}\text { Steatocystoma } \\
\text { multiplex }\end{array}$} \\
\hline & \multirow[b]{2}{*}{ Epidermis } & \multirow[b]{2}{*}{ Infundibulum } & \multirow[b]{2}{*}{ ORS } & \multirow[b]{2}{*}{ Sebaceous gland } & \multirow[b]{2}{*}{ Cyst wall } & \multicolumn{2}{|c|}{ SGLS } & \multirow[b]{2}{*}{ Cyst wall } & \multirow[b]{2}{*}{ SGLS } \\
\hline & & & & & & Sebaceous acinus & Duct like-structure & & \\
\hline CK1 & $+(\mathrm{sb})$ & $+(\mathrm{sb})$ & - & - & $+(\mathrm{sb})$ & - & - & - & - \\
\hline CK7 & - & - & - & - & - & - & - & - & - \\
\hline CK8 & - & - & - & - & - & - & - & - & - \\
\hline CK10 & $+(\mathrm{sb})$ & $+(\mathrm{sb})$ & - & - & $+(\mathrm{sb})$ & - & - & - & - \\
\hline CK14 & $+(\mathrm{sb})$ & $+(\mathrm{b})$ & + & + & +(b) or - & + & - & $+(\mathrm{b})$ & $+(\mathrm{sc})$ \\
\hline CK15 & - & - & - & - & - & - & - & - & - \\
\hline CK16 & - & - & $+(\mathrm{sb})$ & - & - & - & - & - & - \\
\hline CK17 & - & - or + & $+(\mathrm{sb})$ & $+(\mathrm{sdc})$ & - & - & + & + & $+(\mathrm{sdc})$ \\
\hline CK18 & - & - & - & - & - & - & - & - & - \\
\hline CK19 & - & - & - or + & - & - & - & - & - & - \\
\hline Filaggrin & $+(\mathrm{g})$ & $+(\mathrm{g})$ & - & - & $++(g)$ & - & - & ND & ND \\
\hline
\end{tabular}

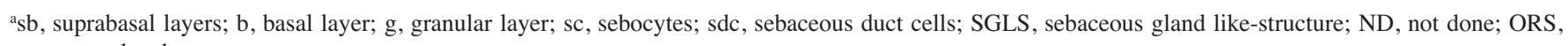
outer root sheath.

layers in the cyst wall, and were not expressed in SGLS. CK14 was partly expressed in basal layer in the cyst wall and sebaceous acinus in SGLS (Fig. 3). CK17 was found only in the sebaceous duct-like structure (Fig. 4). CK7, 8, 15, 16 and 18 were not detected in cyst wall and SGLS. Filaggrin was intensely detected in the granular layer in the cyst wall of DC (Fig. 5), but was not detected in SGLS.

\section{Discussion}

DC is usually a hamartoma which develops in head especially around eyes and neck. Histopathologically, DC is lined by an epidermis with epidermal appendages that are fully matured. In the lumen, hair shafts and keratin debris are commonly observed. 


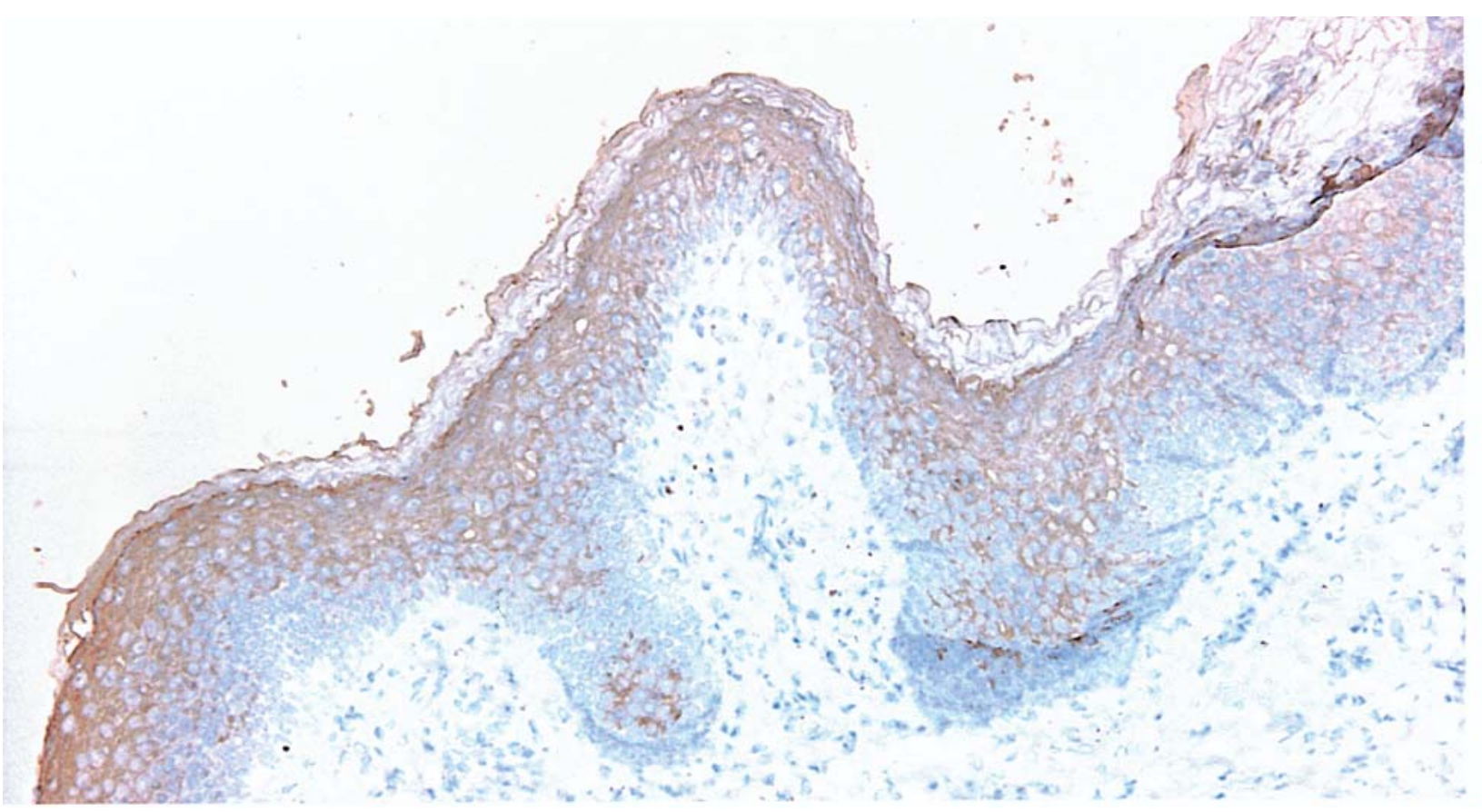

Figure 2. CK1 is expressed in suprabasal layers in the cyst wall (immunohistochemical staining; original magnification x100).

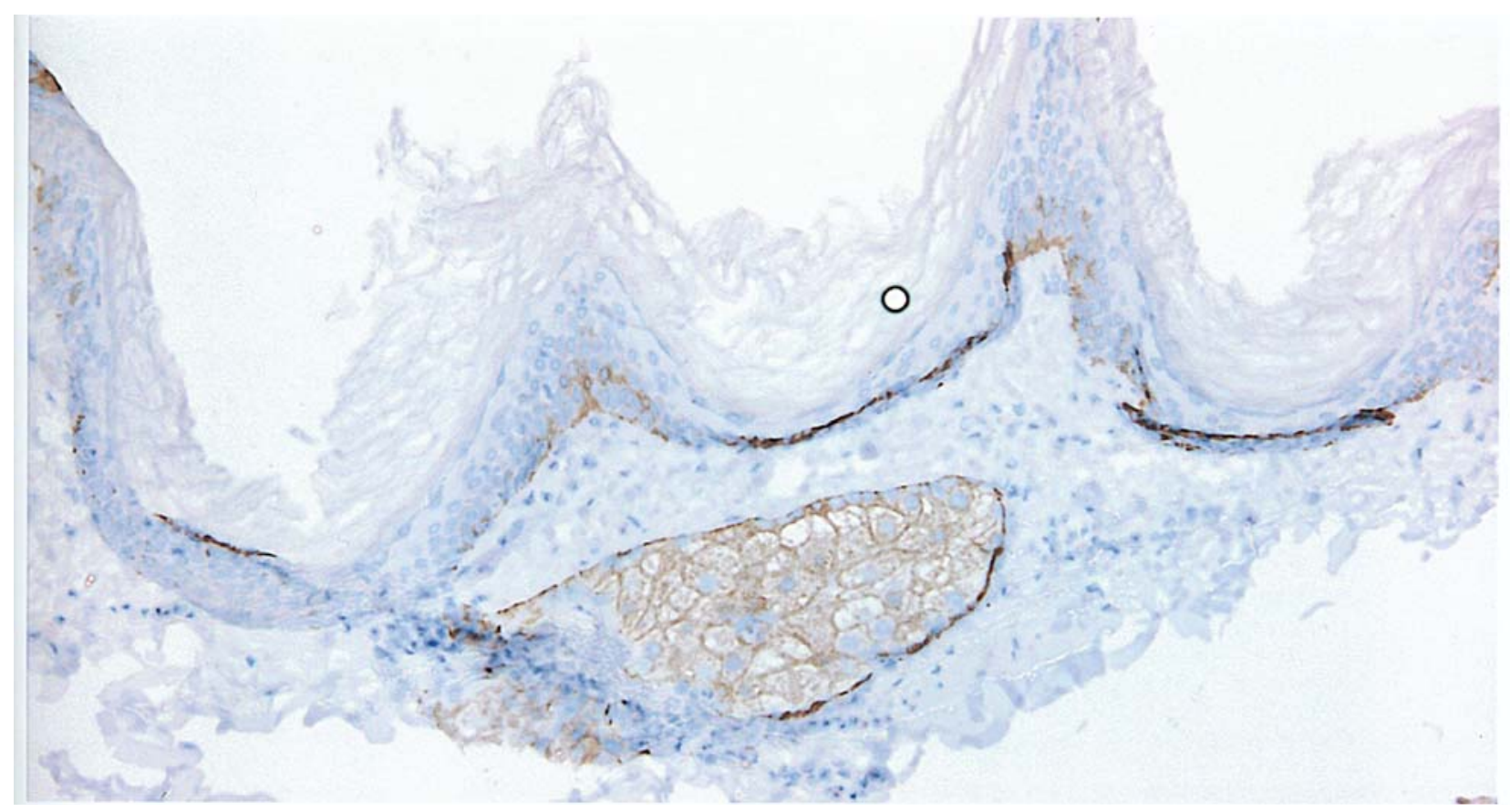

Figure 3. CK14 is partly expressed in basal layer in the cyst wall and sebaceous acinus in SGLS (immunohistochemical staining; original magnification x100).

In the normal pilosebaceous unit, $\mathrm{CK}$ and filaggrin expression has been reported previously (10-12). CK1 and 10 are detected in suprabasal layers in the epidermis and follicular infundibulum. CK14 is expressed in the basal layer in the epidermis and follicular infundibulum, and sebaceous acinus. CK16 is expressed in the outer root sheath beneath the opening of the sebaceous duct. CK17 is detected in the infrainfundibulum and sebaceous duct. Filaggrin is detected in the granular layer and superficial layer in the infundibulum and sebaceous duct.
Cyst wall expressed CK1 and 10 in the suprabasal layers. CK14 expression was limited to the basal layer. CK16 was not detected in the cyst wall. Filaggrin was intensely detected in the granular layer in the cyst wall. In steatocystoma multiplex, cyst wall express CK17, suggesting differentiation towards sebaceous duct (10). CK and filaggrin expression in the cyst wall of DC was similar to that of follicular infundibulum. In the lumen of DC, numerous vellus hairs were observed. These findings suggest that the cyst wall of DC differentiates towards follicular infundibulum. 


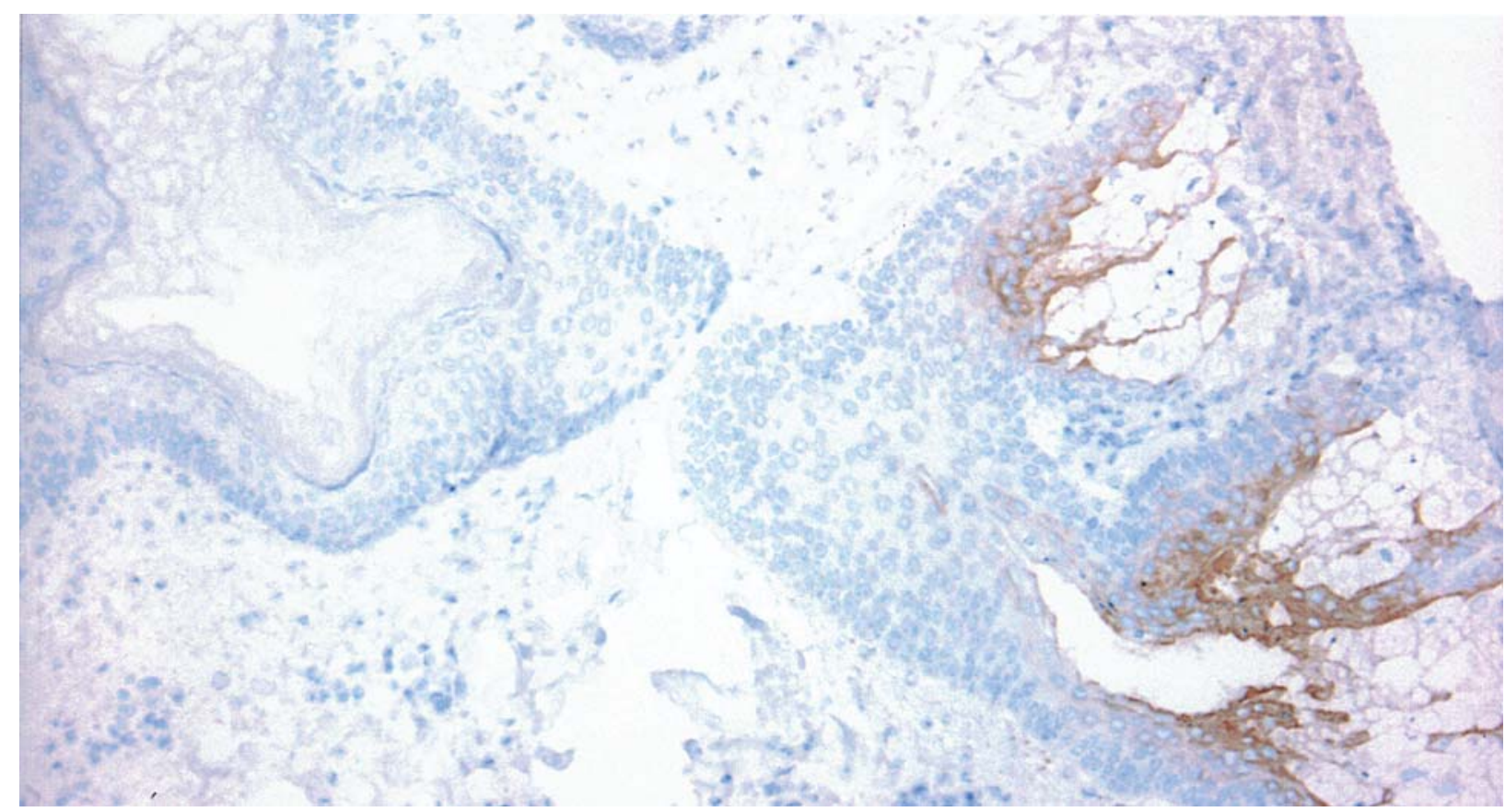

Figure 4. CK17 is found only in the sebaceous duct-like structure (immunohistochemical staining; original magnification x100).

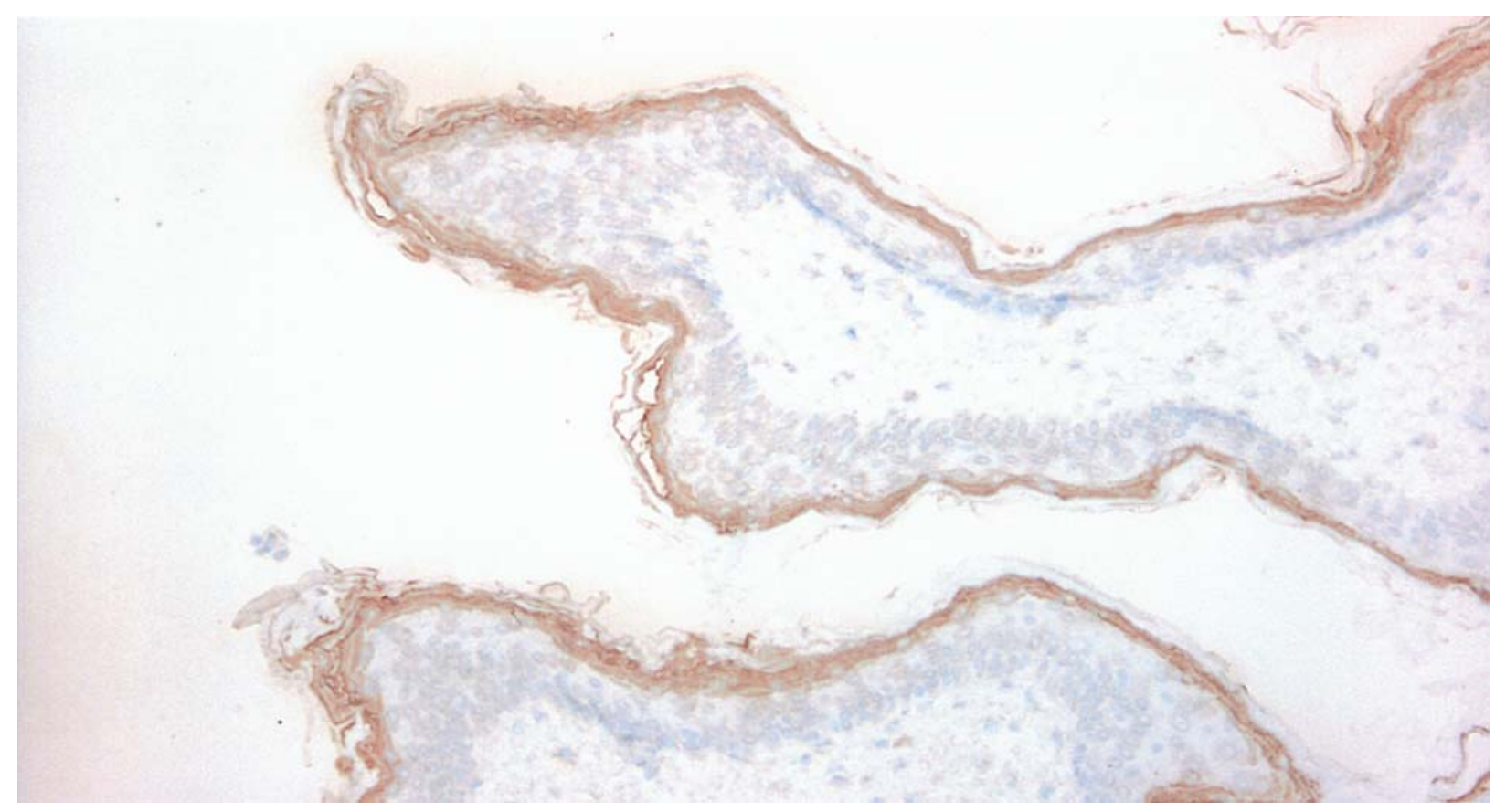

Figure 5. Filaggrin is intensely stained in the granular layer of the DC (immunohistochemical staining; original magnification x100).

In SGLS, CK14 was positive for acinus, and CK17 was positive in the duct-like structure. CK expression of SGLS was similar to that of sebaceous gland, suggesting SLGS in DC differentiates towards the mature sebaceous gland.

CK14 expression was relatively diminished, and CK16 and 17, hyperproliferative keratins, were not found in DC, suggesting that DC has characteristics of a nevoid.

In epidermal cyst, $\mathrm{CK}$ expression is identical to that of follicular infundibulum or normal epidermis (13). In trichilemmal cyst, CK16 is expressed in the cyst wall (13), suggesting that trichilemmal cyst differentiates towards follicular isthmus of the anagen-phase (14). Therefore, CK and filaggrin are useful markers to distinguish DC, epidermal cysts, steatocystoma multiplex and trichilemmal cysts.

Based on CK expression, our study clearly suggests that dermoid cyst differentiates towards follicular infundibulum and the mature sebaceous gland.

\section{References}

1. Brownstein MH and Helwig EB: Subcutaneous dermoid cysts. Arch Dermatol 107: 237-239, 1973. 
2. Crawford R: Dermoid cysts of the scalp. J Pediatr Surg 25: 294-295, 1990.

3. Meagher PJ and Morrison WAM: An unusual presentation of bilateral prominent ears. Br J Plast Surg 54: 366-367, 2001.

4. Moll R, Franke WW, Schiller D, et al: The catalog of human cytokeratins: patterns of expression in normal epithelia, tumors and cultured cells. Cell 31: 11-24, 1982.

5. Dale B, Holbrook KA, Kimball JR, Hoff M and Sun TT: Expression of epidermal keratins and filaggrin during fetal skin development. J Cell Biol 101: 1257-1269, 1985.

6. Markey AC, Lane EB, McDonald DM and Leigh IM: Keratin expression in basal cell carcinoma. Br J Dermatol 126: 154-160, 1992.

7. Lane EB, Bartek J, Purkis PE and Leigh I: Keratin antigens in differentiating skin. Ann NY Acad Sci 455: 241-258, 1995.

8. Jih DM, Lyle S, Elenitsas R, et al: Cytokeratin 15 expression in trichoepithelioma and a subset of basal cell carcinoma suggests they originate from hair follicle stem cells. J Cutan Pathol 26: 113-118, 1999.

9. Lindberg K and Rheinwald JG: Suprabasal 40 kd keratin (K19) expression as an immunohistologic marker of premalignancy in oral epithelium. Am J Pathol 134: 89-98, 1989.
10. Kurokawa I, Nishijima S, Kusumoto K, Suzuki K, Senzaki H, Shikata $\mathrm{N}$ and Tsubura A: Cytokeratin expression in steatocystoma multiplex. Br J Dermatol 146: 534-535, 2002.

11. Kurokawa I, Mayer-da-Silva, Gollnick H and Orfanos CE: Monoclonal antibody labeling for cytokeratins and filaggrin in normal, seborrhoeic and acne skin. J Invest Dermatol 91: 566-571, 1988.

12. Kurokawa I, Nishijima S, Kusumoto K, Senzaki H, Shikata N and Tsubura A: Trichilemmoma: an immunohistochemical study of cytokeratins. Br J Dermatol 149: 99-104, 2003.

13. Ohnishi T and Watanabe S: Immunohistochemical observation of cytokeratins in keratinous cysts including plantar epidermoid cyst. J Cutan Pathol 26: 424-429, 1999.

14. Broekaert D, Goeman L, Ramaekers FC, et al: An investigation of cytokeratin expression in skin epithelial cysts and some uncommon types of cystic tumours using chain-specific antibodies. Arch Dermatol Res 282: 383-391, 1990. 